\title{
ON THE OPTIMUM STRUCTURE OF THE HIERARCHY IN AN ORGANISATION
}

\author{
Ming-Yueh TARNG \\ Instume of Management Science, Chiao-Tung University, Taiwan, R.O.C. \\ Miao-Sheng CHEN \\ Instimute of Management Scrence, Chiao-Tung Liniversity; and Deparment of Mathematics. \\ Tamkang University, Taiwan. R.O.C. \\ Communicated by K.M. Kim \\ Received 3 September 1986 \\ Revised 17 October 1986
}

\begin{abstract}
Using the character of organizations and the elementary results of queueing theory, a yuantitative model of the hierarchy is presented. Its aim is 10 minimize cost, which are the sum of wage costs and costs caused by delays in decision making. With this model, the sensitivily analysis on key variables of the optimum structure is concretely discussed.
\end{abstract}

Key words: Organization design; hierarchy structure; sensitivity analysis.

\section{Introduction}

In the design of organization structure, among the important factors to be considered are the quality, the speed, and the cost of the staff of job planning. For nonhierarchical organization, because there is a lack of a superior-subordinate relationship among the decision makers, the action of planning tends to bias towards the consideration of strategies (Marschak and Radnew, 1972; Nojiri, 1980). For hierarchical organizations, all the above three mentioned factors are important in the design of organization structure (Beckmann, 1960; Williamson, 1967). However, the quality of planning jobs does not have satisfactory measurement tools, therefore, only the speed and the cost of job planning will be considered in this paper. A model is established based on these two factors which will provide the optimum organization structure by considering the trade-off between these two conflicted factors.

Keren and Levhari (1979) try to explain both the existence of hierarchies and their structure by positing that they serve the need to reduce the planning time of the general manager. They assume that the planning time of each level in the hierarchy is linear on the level's span of control, and use the sum of level's planning time to measure the speed of the planning of an organization. Although Keren and Levhari 
gave the topic of the hierarchy structure a general discussion, the following three factors were still ignored in their model: (1) It did not provide us with sufficient reasons that the planning time of a group leader must vary linearly with his span of control. In fact, many authors (Caplow, 1957; Costs and Updegraff, 1973; Pugh and Hickson, 1976; Spyros and Demitris, 1982) on organization theory have suggested that communication interaction, co-oordinations and control problems increase at a faster rate than size. (2) It did not convince us with sufficient reasons that the planning time of a group leader depends on his span of control and independs on the level where he lies. In fact, since the upper level of group leaders must bear more uncertainties for decision making, so, with the same span of control, the planning time of group leaders in the upper level is usually greater than that of group leaders in the lower level (Starbuck, 1979). (3) It only uses the proceeding time of a planning job to measure the speed of the planning, and omit its waiting time. if a new job occurs and all group leaders have not finished their own tasks for primary jobs, then this new job may be postponed for planning in the hierarchy. Additionally, even though the new job could be planning immediately in the hierarchy, the waiting time may still exist in some levels if the level's planning times are not all equal.

In this paper we shall use the results of queueing theory to formulate the waiting time of a job, and then present a general model to discuss the sensitivity analysis on optimum structure variables - the height of the hierarchy, the number of group leaders in the hierarchy, the level's span of control, and the idle time of a group leader; with respect to the parameters - the wage rate, the organization size, the complexities of planning jobs, and the expected interarrival times of planning jobs, separately.

\section{Assumptions and notations}

The organization studied in this model is composed of (fundamental) activity units which are completely dependent on the head, or general manager, for instructions. The most crucial topics for the head, is to seek a hierarchy structure which can quickly and effectively solve the problems that arise during the execution of the plan of action. For this purpose, the head has to prepare a new set of instructions to coordinate these activity units on the basis of new observations made by them. To shorten the time it takes to collect the information and prepare the instructions, the head has to interpose additional levels of the hierarchy between himself and activity units. The hierarchy of the organization is populated by identical group leaders, which links head and activity units. The wage rate per group leader is denoted by w.

The main contribution of the group leader is to find the relationship among his (immediate) subordinate reportings, and transmit it to his (immediate) superior. To insure the sum of group leader's planning times that appear in a path directed from 
any activity unit to the head, are all equal; the complexities of tasks and the span of control of all group leaders in a given level, will be the same.

\subsection{Structure variables}

$n=$ the number of activity units; we refer to $n$ as the size of an organization.

$H=$ the number of hierarchy levels; we refer to $H$ as the height of the hierarchy.

$x_{h}=$ the number of group leaders in level $h, x_{1}=1$ and $x_{H+1}=n$.

$s_{h}=x_{h+1} / x_{h}$, is the span of control of a group leader in level $h$.

$M=x_{1}+x_{2}+\cdots+x_{H}$, is the number of group leaders in the whole hierarchy.

In our model, $x_{h}$ and hence $s_{h}$ are considered as continuous variables; this consideration is reasonable in the real word, because the measurement of $x_{h}$ is based on the time which is spent in planning during a day.

\subsection{The contribution of group leaders}

$a=$ the index of complexities of an activity unit's reporting (with respect to a planning job); we refer to $a \cdot n$ as the index of the initial complexities of a planning job.

$b \cdot a \cdot n=$ the index of the final complexities of a planning job as it is finished by group leaders, where $0<b<1$.

$z_{h}=$ the index of complexities of an $h$-level group leader's reporting, $z_{1}=b \cdot a \cdot n$ and $z_{H+1}=a$.

$c\left(G_{h}\right)=$ the contribution of a $h$-level group leader $G_{h}$,

$=\operatorname{Input}\left(G_{h}\right)-\operatorname{Output}\left(G_{h}\right)$, $=s_{h} z_{h+1}-z_{h}$.

$f\left(c\left(G_{h}\right)\right)=$ the planning time required by $G_{h}$ to complete his own task.

Graciunas (1937) presented a mathematical model to demonstrate how the complexities of superior-subordinate potential interacts. His formula states that as the number of subordinates reporting to a group leader increases arithmetically, the number of potential interactions increases geometrically. This means that $f$ have the following properties:

$$
f>0, \quad f^{\prime}>0 \text { and } f^{\prime \prime} \geq 0 .
$$

In general, the group leaders in the upper levels must bear more uncertainties in the planning due to the increasing complexity that exists in their subordinated reportings. Hence the assumption we make here is

$$
z_{h} / z_{h+1}=\theta, \quad \theta \geq 1 \text { is a parameter. }
$$

In the hierarchy, the more information that is missing means the increasing final complexity of a planning job (i.e. $b$ increasing). There are two aspects about the missing information. In the longitudinal aspect, it usually depends on the superior-subordinate communications. In the cross section aspect, the missing information is usually caused by the delivery of group leader's tasks. Given In- 
$\operatorname{put}\left(G_{h}\right)$, the smaller the value of $\theta$ implies that the more detailed data's statement on facts, could be found in $G_{h}$ 's reporting. Additionally, with the same height $H$. the decrease of $\theta$ yields the decrease of the final complexities of a planning job (ct. (3.1)).

\subsection{The waiting time of a planning job}

Consider the hicrarchy as a queueing system (regarding planning jobs and group leaders as customers and severs respectively). If we assume that this queueing system has Poisson input process with mean interarrival times e, and it has constant service times; then the expected waiting time of a planning job (Hillier and Lieberman, 1980, p. 437) is given by

$$
\text { w.t. }=\frac{t^{2}}{2(e-t)}, \quad \text { where } t=\max _{1 \leq h \leq H} f\left(c\left(G_{h}\right)\right) \text {. }
$$

\subsection{The proceeding time of a planning job}

In general, group leaders in the same level of a hierarchy are working concurrently, and group leaders of immediately superior (or inferior) level wait until the adjacent level has finished. So the proceeding time of a planning job is defined by

$$
\text { p.t. }=\sum_{h=1}^{H} f\left(c\left(G_{h}\right)\right) \text {. }
$$

\subsection{Costs caused by delays in decision making}

The cost of per unit time profits lost through slow planning, is defined by

$$
C(T)=C(\text { w.t. + p.t. }), \text { where } C^{\prime}(T)>0 \text { and } C^{\prime \prime}(T) \geq 0 \text {. }
$$

\section{The model of a hierarchy}

Assumption (2.2) yields that

$$
z_{h}=\theta z_{h+1}=\cdots-\theta^{H-h+1} z_{h / 1}=\theta^{H-h+!} a
$$

and therefore we have the following properties:

$$
\begin{aligned}
& b \cdot a \cdot n=z_{1}=\theta^{H} a, \\
& c\left(G_{h}\right)=\left(s_{h}-\theta\right) z_{h+1}=\left(s_{h}-\theta\right) \theta^{-h} b \cdot a \cdot n, \\
& \text { p.t. }=\sum_{h=1}^{H} f\left(\left(s_{h}-\theta\right) \theta^{-h} b \cdot a \cdot n\right) .
\end{aligned}
$$


The objective of this model is to minimize the total cost L, which are the sum of wage costs and costs caused by delays in decision making. That is

$$
\text { (I) }\left\{\begin{array}{l}
\min L=C(\text { w.t. }+ \text { p.t. })+\text { w. }\left(\sum_{h=1}^{H} x_{h}\right) \\
\text { subject to: } x_{1}=1, x_{H+1}=n, x_{h+1}=s_{h} x_{h}, 1 \leq h \leq H \\
\theta^{H}=b \cdot n, \text { p.t. }=\sum_{h=1}^{H} f\left(\left(s_{h}-\theta\right) \theta^{-h} b \cdot a \cdot n\right), \text { w.t. }=\frac{i}{2(e-n)} \\
\text { where } t=\max _{1 \leq h \leq H} f\left(\left(s_{h}-\theta\right) \theta^{-h} b \cdot a \cdot n\right),
\end{array}\right.
$$

where $a, n, w, e, \theta \geq 1,0<b<1$, are parameters.

Model ( 1 ) is a generalization of the Keren and Levhari model. If we omit the term w.1., restrict $f$ to be a linear function and set $\theta=1$; then it is the Keren and Levhari model.

\section{The optimum solution of the hierarchy model}

Let

$$
l_{h}=f\left(\left(s_{h}-\theta\right) \theta^{-h} b \cdot a \cdot n\right), \quad 1 \leq h \leq H .
$$

It is valid that

$$
\frac{\mathrm{d}(\mathrm{w} . \mathrm{t} .)}{\mathrm{dt}}=\frac{\mathrm{d}}{\mathrm{d} t}\left(\frac{t^{2}}{2(e-t)}\right)=\frac{t(2 e-t)}{2(e-t)^{2}}>0
$$

and therefore w.t. increases with $t$. This implies that, for fixed p.t. $=\sum t_{n}$, the necessary condition of the minimization of w.t. is

$$
t=t_{1}=t_{2}=\cdots=t_{H}
$$

Add this condition in Model (I), and it leads to the following properties:

$$
\begin{aligned}
v=( & \left.S_{1}-\theta\right) \theta^{-1} b \cdot a \cdot n=\left(s_{2}-\theta\right) \theta^{-2} b \cdot a \cdot n=\cdots \\
= & \left(s_{H}-\theta\right) \theta^{-H} b \cdot a \cdot n, \\
v \cdot M= & v\left(x_{1}+x_{2}+\cdots+x_{H}\right), \\
= & \left(s_{1}-\theta\right) \theta^{-1} b \cdot a \cdot n \cdot x_{1}+\left(s_{2}-\theta\right) \theta^{-2} b \cdot a \cdot n \cdot x_{2}+\cdots \\
& +\left(s_{H}-\theta\right) \theta^{-H} b \cdot a \cdot n \cdot x_{H}, \\
= & b \cdot a \cdot n\left[\left(x_{2} \theta^{-1}+x_{3} \theta^{-2}+\cdots+x_{H+1} \theta^{-H}\right)-\left(x_{1}+x_{2} \theta\right.\right. \\
& \left.\left.+\cdots+x_{H} \theta^{-H+1}\right)\right] ; \text { by }(3.1), \\
= & (1-b) a \cdot n,
\end{aligned}
$$




$$
\begin{aligned}
\text { p.t. } & =H f(u) ; \text { by }(3.1), \\
& =\frac{\ln b+\ln n}{\ln \theta}-f(v) .
\end{aligned}
$$

Therefore Model (I) can be written as the following form

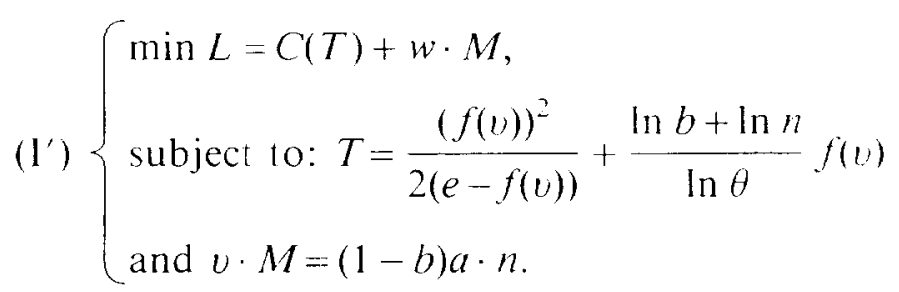

The necessary condition of this optimality is

$$
\mathrm{d} L / \mathrm{d} M=-C^{\prime}(T) T^{\prime}(v)(1-b) a \cdot n \cdot M^{-2}+w=0 .
$$

\section{The sensitivity analysis of the optimum solution}

The total differentiation of (4.5) yields

$$
\frac{\mathrm{d} w}{w}=\frac{C^{\prime \prime}(T)}{C^{\prime}(T)} \mathrm{d} T+\frac{T^{\prime \prime}(v)}{T^{\prime}(v)} \mathrm{d} v-\frac{\mathrm{d} b}{1-b}+\frac{\mathrm{d} a}{a}+\frac{\mathrm{d} n}{n}-\frac{2 \mathrm{~d} M}{M} .
$$

The total differentiation of (4.3) yields

$$
\mathrm{d} T=\frac{\partial T}{\partial v} \mathrm{~d} v+\frac{\partial T}{\partial b} \mathrm{~d} b+\frac{\partial T}{\partial n} \mathrm{~d} n+\frac{\partial T}{\partial \theta}+\frac{\partial T}{\partial e} \mathrm{~d} e .
$$

The total differentiation of (4.4) yields

$$
M \cdot \mathrm{d} v=(1-b)(a \cdot \mathrm{d} n+n \cdot \mathrm{d} a)-a \cdot n \cdot \mathrm{d} b-\frac{(1-b) \cdot a \cdot n}{M} \mathrm{~d} M .
$$

Substituting (5.2) and (5.3) in (5.1), leads to

$$
\begin{aligned}
\frac{\mathrm{d} w}{w}= & -\left[\frac{u(1-b) \cdot a \cdot n}{M^{2}}+\frac{2}{M}\right] \mathrm{d} M+\frac{C^{\prime \prime}(T)}{C^{\prime}(T)} \frac{\partial T}{\partial \theta} \mathrm{d} \theta \\
& +\left[\frac{u \cdot a \cdot(1-b)}{M}+\frac{C^{\prime \prime}(T)}{C^{\prime}(T)} \frac{\partial T}{\partial n}+\frac{1}{n}\right] \mathrm{d} n \\
& -\left[\frac{u \cdot a \cdot n}{M}-\frac{C^{\prime \prime}(T)}{C^{\prime}(T)} \frac{\partial T}{\partial b}+\frac{1}{1-b}\right] \mathrm{d} b \\
& +\left[\frac{u \cdot n}{M}-\frac{1}{a}\right] \mathrm{d} a+\frac{C^{\prime \prime}(T)}{C^{\prime}(T)} \frac{\partial T}{\partial e} \mathrm{~d} e,
\end{aligned}
$$

where 


$$
u=\frac{C^{\prime \prime}(T)}{C^{\prime}(T)} T^{\prime}(v)+\frac{T^{\prime \prime}(v)}{T^{\prime}(v)}, \quad\left(T^{\prime}(v) \text { means } \frac{\partial T}{\partial v}\right)
$$

From (4.3), it can be shown that

$$
\begin{aligned}
T^{\prime}(v) & =\frac{f(u) f^{\prime}(v)(2 e-f(v))}{2(e-f(v))^{2}}+\frac{\ln b+\ln n}{\ln \theta} f^{\prime}(v)>0 \\
T^{\prime \prime}(v) & =\frac{f(v) f^{\prime}(v)(2 e-f(v))}{2(e-f(v))^{2}}\left[\frac{f^{\prime}(v)}{f(v)}+\frac{f^{\prime \prime}(u)}{f^{\prime}(u)}+f^{\prime}(v)\left(\frac{2}{e-f(u)}-\frac{1}{2 e-f(v)}\right) \mid\right. \\
& +\frac{\ln b+\ln n}{\ln \theta}-f^{\prime \prime}(v)>0 .
\end{aligned}
$$

This implies $u>0$.

\subsection{The effect of changing the wage rate $w$}

Theorem. In the hierarchy model, if we consider $w$ as a variable and keep other parameters fixed; then

$$
\begin{aligned}
& \frac{\mathrm{d} M}{\mathrm{~d} w}=-\left[w\left(\frac{u(1-b) \cdot a \cdot n}{M^{2}}+\frac{2}{M}\right)\right]^{-1}<0, \\
& \frac{\mathrm{d} u}{\mathrm{~d} w}=-\frac{(1-b) \cdot a \cdot n}{M^{2}} \frac{\mathrm{d} M}{\mathrm{~d} w}>0, \\
& \frac{\mathrm{d} s_{h}}{\mathrm{~d} w}=-\frac{\theta^{h}(1-b)}{b \cdot M^{2}} \frac{\mathrm{d} M}{\mathrm{~d} w}>0, \\
& \frac{\mathrm{d} I}{\mathrm{~d} w}=\frac{f^{\prime}(v)(1-b) \cdot a \cdot n}{M^{2}} \frac{\mathrm{d} M}{\mathrm{~d} w}<0,
\end{aligned}
$$

where $I=e-f(v)$ is the idle time of a group leader.

Proof. (1) By setting $\mathrm{d} n=\mathrm{d} e=\mathrm{d} a=\mathrm{d} b=\mathrm{d} \theta=0$ in (5.4).

(2) By differentiating (4.2): $v=(1-b) \cdot a \cdot n \cdot M^{-1}$ with respect to $w$.

(3) By differentiating (4.1): $v=\left(s_{h}-\theta\right) \theta^{-h} b \cdot a \cdot n$ with respect to $w$. and using the result of $\mathrm{d} u / \mathrm{d} w$.

(4) By differentiating: $I=e-f(v)$ with respect to $w$, and using the result of $\mathrm{d} v / \mathrm{d} w$.

\subsection{The effect of changing the organization size $n$}

Theorem. In the hierarchy model, if we consider $n$ as a variable and keep other parameters fixed; then

$$
\frac{\mathrm{d} M}{\mathrm{~d} n}=\left[\frac{u(1-b) \cdot a}{M}+\frac{C^{\prime \prime}(T)}{C^{\prime}(T)} \cdot \frac{f(v)}{n \ln \theta}+\frac{1}{n}\right]\left[\frac{u(1-b) \cdot a \cdot n}{M^{2}}+\frac{2}{M}\right]^{-1}>0
$$


(2)

$$
\begin{aligned}
& \frac{\mathrm{d} s_{h}}{\mathrm{~d} n}=-\frac{(1-b) \theta^{h}}{b \cdot M^{2}} \frac{\mathrm{d} M}{\mathrm{~d} n}<0, \\
& \frac{\mathrm{d} H}{\mathrm{~d} n}=\frac{1}{n \ln \theta}>0, \\
& \frac{\mathrm{d} I}{\mathrm{~d} n}=\frac{f^{\prime}(u)(1-b) \cdot a}{M^{2}}\left(M-n \cdot \frac{\mathrm{d} M}{\mathrm{~d} n}\right) .
\end{aligned}
$$

Proof. (1) By setting $\mathrm{d} w=\mathrm{d} e=\mathrm{d} a=\mathrm{d} b=\mathrm{d} \theta=0$ in (5.4), and using the differentiation of (4.3) with respect to $n$.

(2) Together with the differentiation of (4.1):

$$
\frac{\mathrm{d} v}{\mathrm{~d} n}=\theta^{-h} b \cdot a \cdot n \cdot \frac{\mathrm{d} s_{h}}{\mathrm{~d} n}+\frac{v}{n}=\theta^{-h} b \cdot a \cdot n \cdot \frac{\mathrm{d} s_{h}}{\mathrm{~d} n}+\frac{(1-b) \cdot a}{M}
$$

and the differentiation of (4.2):

$$
\frac{\mathrm{d} v}{\mathrm{~d} n}=\frac{(1-b) \cdot a}{M^{2}}\left(M-n \frac{\mathrm{d} M}{\mathrm{~d} n}\right) .
$$

(3) By differentiating (3.1) with respect to $n$.

(4) By differentiatiang idle time: $I=e-f(v)$ with respect to $n$, and using (5.5).

Remark. A simple computation yields that $\mathrm{d} I / \mathrm{d} n \geq 0$ if and only if $C^{\prime \prime}(T) f(v) \geq$ $C^{\prime}(T) \ln \theta$. In particular if $C$ is a linear function then $\mathrm{d} I / \mathrm{d} n<0$.

\subsection{The effect of changing the mean interarrival time $e$}

Theorem. In the hierarchy model, if we consider $e$ as a variable and keep other parameters fixed; then

$$
\begin{aligned}
& \frac{\mathrm{d} M}{\mathrm{~d} e}=-\frac{C^{\prime \prime}(T)}{C^{\prime}(T)} \frac{f^{2}(v)}{(e-f(v))^{2}} \frac{2 M^{2}}{u(1-b) \cdot a \cdot n+2 M} \leq 0, \\
& \frac{\mathrm{d} s_{h}}{\mathrm{~d} e}=-\frac{(1-b) \cdot a \cdot n \cdot \theta^{h}}{a \cdot n \cdot M^{2} \cdot b} \frac{\mathrm{d} M}{\mathrm{~d} e} \geq 0, \\
& \frac{\mathrm{d} I}{\mathrm{~d} e}=1-\frac{f^{\prime}(u)(1-b) \cdot a \cdot n}{M^{2}} \frac{\mathrm{d} M}{\mathrm{~d} e} .
\end{aligned}
$$

Proof. (1) By setting $\mathrm{d} w=\mathrm{d} a=\mathrm{d} n=\mathrm{d} b=\mathrm{d} \theta=0$ in (5.4), and using the differentiation of (4.3) with respect to $e$.

(2) Together with the differentiation of (4.1) and the differentiation of (4.2) (with respect to $e$ ).

(3) Together with the differentiation of the equation: $I=e-f(u)$ and the differentiation of (4.2). 
Remark. A simple computation yields that if $C$ is a linear function then $\mathrm{d} M / \mathrm{d} e=0$ and $\mathrm{d} I / \mathrm{d} e=1$.

\subsection{The effect of changing the initial complexities a}

Theorem. In the hierarchy model, if we consider a as a variable and keep other parameters fixed; then

$$
\begin{aligned}
& \frac{\mathrm{d} M}{\mathrm{~d} a}=\left[\frac{u \cdot n}{M}+\frac{1}{a}\right]\left[\frac{u(1-b) \cdot a \cdot n}{M^{2}}+\frac{2}{M}\right]^{-1}>0, \\
& \frac{\mathrm{d} s_{h}}{\mathrm{~d} a}=-\frac{1-b}{b \cdot M^{2}} \theta^{h} \frac{\mathrm{d} M}{\mathrm{~d} a}<0, \\
& \frac{\mathrm{d} I}{\mathrm{~d} a}=\frac{f^{\prime}(u)(1-b) \cdot n \cdot(u \cdot b \cdot a \cdot n-M)}{M(u(1-b) \cdot a \cdot n+2 M)} ; \text { and } \frac{\mathrm{d} I}{\mathrm{~d} a}>0 \text { iff } u \cdot b \cdot a \cdot n>M
\end{aligned}
$$

Proof. (1) By setting $\mathrm{d} w=\mathrm{d} e=\mathrm{d} n=\mathrm{d} b=\mathrm{d} \theta=0$ in (5.4).

(2) Together with the differentiation of (4.1):

$$
\frac{\mathrm{d} v}{\mathrm{~d} a}=\theta^{-h} b \cdot a \cdot n \frac{\mathrm{d} s_{h}}{\mathrm{~d} a}+\frac{v}{a}=\theta^{-l} b \cdot a \cdot n \frac{\mathrm{d} s_{h}}{\mathrm{~d} a}+\frac{(1-b) \cdot n}{m}
$$

and the differentiation of (4.2):

$$
\frac{\mathrm{d} v}{\mathrm{~d} a}=\frac{(1-b) \cdot n}{M^{2}}\left(M-a \frac{\mathrm{d} M}{\mathrm{~d} a}\right),
$$

$$
\begin{aligned}
\frac{\mathrm{d} I}{\mathrm{~d} a} & =-f^{\prime}(u) \frac{\mathrm{d} u}{\mathrm{~d} a} ; \text { by }(5.6) \\
& =\frac{f^{\prime}(u)(1-b) \cdot n}{M^{2}}\left(a \frac{\mathrm{d} M}{\mathrm{~d} a}-M\right)
\end{aligned}
$$

and then by using the result of $\mathrm{d} M / \mathrm{d} a$.

\subsection{The effect of changing the ratio of complexities $\theta$}

Theorem. In the hierarchy model, if we consider $\theta$ as a variable and keep other parameters fixed; then

$$
\begin{aligned}
& \frac{\mathrm{d} M}{\mathrm{~d} \theta}=-\frac{M^{2} f(u)}{u(1-b) \cdot a \cdot n+2 M} \frac{C^{\prime \prime}(T)}{C^{\prime}(T)} \frac{\ln b+\ln n}{\theta(\ln \theta)^{2}} \leq 0, \\
& \frac{\mathrm{d} s_{h 2}}{\mathrm{~d} \theta}=1+(b \cdot a \cdot n)^{-1}\left|h \cdot v \cdot \theta^{h-1}-\frac{(1-b) \cdot a \cdot n \cdot \theta^{h}}{M^{2}} \frac{\mathrm{d} M}{\mathrm{~d} \theta}\right|>0,
\end{aligned}
$$$$
\frac{\mathrm{d} H}{\mathrm{~d} \theta}=-\frac{\ln b+\ln n}{\theta(\ln \theta)^{2}}<0,
$$ 


$$
\frac{\mathrm{d} I}{\mathrm{~d} \theta}=\frac{f^{\prime}(u)(1-b) \cdot a \cdot n}{M^{2}} \frac{\mathrm{d} M}{\mathrm{~d} \theta} \leq 0 .
$$

Proof. (1) By setting $\mathrm{d} w=\mathrm{d} a=\mathrm{d} n=\mathrm{d} b=\mathrm{d} e=0$ in (5.4), and using the differentiation of (4.3) with respect to $\theta$.

(2) Together with the differentiation of (4.1) and the differentation of (4.2) (with respect to $\theta$ ).

(3) By differentiating (3.1) with respect to $\theta$.

(4) Together with the differentiation of the equation: $l=e-f(0)$ and the differentiation of $(4.2)$ (with respect to $\theta$ ).

\subsection{The effect of changing the final complexities $b$}

Theorem. In the hierarchy model, if we consider $b$ as a variable and keep other parameters fixed; then

$$
\begin{aligned}
& \frac{\mathrm{d} M}{\mathrm{~d} b}=-\left[\frac{u \cdot a \cdot n}{M}-\frac{C^{\prime \prime}(T)}{C^{\prime}(T)} \frac{f(u)}{b \ln \theta}+\frac{1}{1-b}\right]\left[\frac{u(1-b) \cdot a \cdot n}{M^{2}}+\frac{2}{M}\right]^{-1}, \\
& \frac{\mathrm{d} s_{h}}{\mathrm{~d} b}=-\frac{\theta^{h}}{(b M)^{2}}\left[(1-b) \cdot b \frac{\mathrm{d} M}{\mathrm{~d} b}+M\right] .
\end{aligned}
$$

$$
\frac{\mathrm{d} H}{\mathrm{~d} b}=\frac{1}{b \ln \theta}>0
$$

$$
\frac{\mathrm{d} I}{\mathrm{~d} b}=f^{\prime}(u)\left[\frac{a \cdot n}{M}+\frac{(1-b) \cdot a \cdot n \mathrm{~d} M}{M^{2}} \frac{\mathrm{d} b}{\mathrm{~d} b}\right] .
$$

Proof. (1) By setting $\mathrm{d} w=\mathrm{d} a=\mathrm{d} n=\mathrm{d} \theta=\mathrm{d} e=0$ in (5.4), and using the differentiation of (4.3) with respect to $b$.

(2) Together with the differentiation of (4.1) and the differentiation of (4.2) (with respect to $b$ ).

(3) By differentiating (3.1) with respect to $b$.

(4) Together with the differentiation of the equation: $l=e-f(u)$ and the differentiation of (4.2) (with respect to $b$ ).

Remark. (I) If $C$ is a linear function, then $\mathrm{d} M / \mathrm{d} b<0$. (II) If $\mathrm{d} M / \mathrm{d} b>0$, then $\mathrm{d} s_{h} / \mathrm{d} b<0$ and $\mathrm{d} I / \mathrm{d} b>0$.

\section{Conclusions}

The model presented in this paper discusses the uniting of the fundamental activiy units in an organiation, to obtain coordination between the speed of the planning 
jobs and the cost of staffing. Under these assumptions, a quantitative model of the hierarchy structure could be formed.

We have discussed the sensitivity analysis of the optimum structure, and obtained many interesting properties: 11 If the wage rate increases then so does the span of control of a given level, while both the idle time of a group leader and the number of group leaders (in the whole hierarchy) decreases. (ct. Section 5.1). (2) If the organization size increases then so does the number of group leaders and the height of the hierarchy, while the span of control of a given level decreases: and the idle time of a group leader decreases if the indirect cost function is a linear function. (c.t. Section 5.2). (3) If the mean interarrival time of planning jobs increases, then so does the span of control of a given level, while the number of group leaders decreases; and the idle time of a group leader increases if the indirect cost function is a linear function. (cf. Section 5.3). (4) If the initial complexities of planning jobs increase then so does the number of group leaders, while the span of control of a given level decreases. (cf. Section 5.4). (5) If the ratio between the complexities of wo immediately superior-subordinate reportings increases, then so does the span of control of a given level, while the number of group leaders, the height of the hierarchy and the idle time of a group leader all decrease. (cf. Section 5.5). (6) If the final complexities of a planning job as it is finished by group leaders increase, then so does the height of the hierarchy, while the number of group leaders decreases if the indirect cost function is a linear function. (ct. Section 5.6).

Finally we are obliged to remark that this model is still incomplete in the following respects: It ignores the question of how the organization should be classified, and the difference in the wage rates in different levels of the hierarchy. These consideralions are important for the implementation of the theoretical results to the real situation, which are valuable questions to be studied further.

\section{References}

M.1. Beckmann, Some aspects of returns to scale in business administration. (uart. 1. Econom. 7.4 (1960) $464-471$.

T. Caplow, Organizational size, Admin. Science Quart. 1 (1957) 484-505.

R. Costs and D. Updegraff, The relationship between organization size and the admunistrative component of banks, $J$. Business 46 (1973) 576-588.

V.A. Craicunas, Relationship in organization, in: L. Gulick and L. Urwick, eds.. Papers on the Science of Administration (Institute of Public Administration, New York) pp. 181-15i.

1..S. Hillier and Ci.J. Lieberman, Introduction 10 Operations Research (Holder-Das, Inc. San Francisco. 1980).

M. Keren and D. Levhari, The Optimum span of control in a pure hierarchy, Management Science 25. 11 (1979) 1162-1172.

1. Marschak and R. Radnew, Economic Theory of Teams (Yale Univ. Press, New Haven and London, 1472).

14. Nojiri, On the fuzzy team decision in a changing entironment, Fuzzy Sets and Systems 3 (1980) $13 \div-150$ 
D. Pugh and D. Hickson, Organization Structure in its Context (Saxon House, England, 1976).

K. Spyros and A. Demitris, Size and administrative intensity in organizational divisions. Management Science 28, 8 (1982) 854-868.

W.H. Starbuck, Organizational growth and development, in: W.H. Starbuck, ed, Organization Growth and Development (Penguin Books, Harmondsworth, 1979).

O. E. Williamson, Hierarchical control and optimum firm size, J. Political Economy, 75, 2 (1967). 